\title{
COVID-19: recomendaciones generales y especificaciones para el paciente con cáncer de pulmón avanzado. Revisión de la literatura
}

\section{COVID-19: general recommendations and specifications for the patient with advanced lung cancer. Literature review}

\author{
Carla Paola Sánchez-Ríos,* Miguel Ángel Ramírez-Candelas,* Sergio Alejandro Menchaca-Dávila, \\ Luis Manuel Martínez-Barrera,* Jerónimo Rafael Rodríguez-Cid,* Jorge Arturo Alatorre-Alexander*
}

*Instituto Nacional de Enfermedades Respiratorias Ismael Cosío Villegas, Ciudad de México.

RESUMEN. El brote de la nueva enfermedad por coronavirus 2019 (COVID-19) se ha convertido en una amenaza para la salud mundial. Sabemos que el riesgo de enfermedad grave y muerte en los casos de COVID-19 aumenta con la edad y la presencia de condiciones de salud comórbidas como obesidad, hipertensión arterial sistémica, diabetes mellitus, enfermedad pulmonar obstructiva crónica y estados de inmunosupresión como el cáncer. Desde la aparición del primer caso en Wuhan, China, en diciembre de 2019, se han llevado a cabo enormes esfuerzos de investigación para comprender los mecanismos de transmisibilidad y potenciales ofertas terapéuticas que impacten en las tasas de mortalidad. En esta revisión se plantean los potenciales desafíos del paciente infectado por COVID-19 y el cáncer pulmonar.

Palabras clave: COVID19, cáncer, pronóstico.
ABSTRACT. The outbreak of the new coronavirus disease 2019 (COVID-19) has become a threat to global health. We know that the risk of serious disease and death in COVID-19 cases increases with age and the presence of comorbid health conditions such as obesity, systemic arterial hypertension, diabetes mellitus, COPD, and immunosuppressed states such as cancer. Since the first case emerged in Wuhan, China in December 2019, huge research efforts have been made to understand the transmissibility mechanisms and potential therapeutic offerings that impact mortality rates. In this review, the potential challenges of the COVID-19 patient and lung cancer are raised.

Keywords: COVID19, cancer, prognosis.

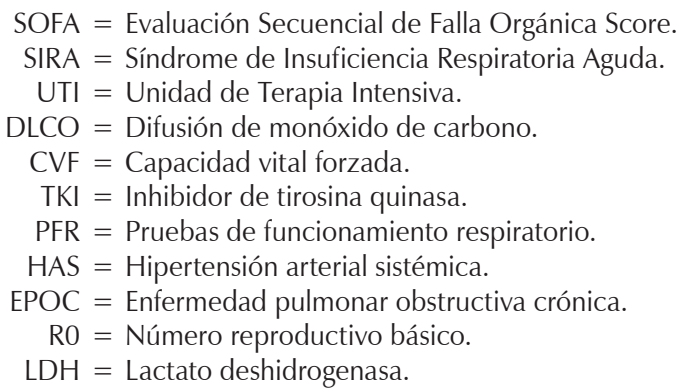

\section{INTRODUCCIÓN}

Estimaciones de la Organización Mundial de la Salud (OMS) han identificado que las enfermedades virales representan un desafío para la salud pública, pues tienen un elevado potencial pandémico. Se tiene evidencia de que en las últimas dos décadas se han registrado varias epidemias virales.

El pasado 31 de diciembre de 2019 se informó por primera vez a la oficina de País de la OMS, en China, un brote de casos con infecciones respiratorias bajas sin agente
Dra. Carla Paola Sánchez Ríos

Instituto Nacional de Enfermedades Respiratorias Ismael Cosío

Villegas, Ciudad de México.

Correo electrónico: pao1144tost@gmail.com

Trabajo recibido: 07-IV-2020; aceptado: 12-V-2020. 
causal identificado. Los primeros casos se clasificaron como «neumonía de etiología desconocida». El Centro Chino para el Control y Prevención de Enfermedades (CDC) y los CDC locales organizaron un programa intensivo de investigación de brotes para su adecuada identificación; al día de hoy, sabemos que esta enfermedad se atribuye a un nuevo virus perteneciente a la familia del coronavirus (CoV) SARS-CoV-2.

Esta infección se ha esparcido rápidamente por todo el mundo, y desde el 30 de enero de 2020 el brote fue declarado por la OMS como emergencia de salud pública de preocupación internacional (PHEIC), pues se había extendido, en ese momento, a 18 países, con cuatro países que habían confirmado la transmisión entre seres humanos. Un punto de inflexión adicional ocurrió el 26 de febrero de 2020, ya que el primer caso de la enfermedad no importado de China se registró en los Estados Unidos. Posteriormente el 28 de febrero se reportó el primer caso confirmado serológicamente en nuestro país, momento desde el cual la cifra ha ido en aumento, cobrando varias vidas.

Las organizaciones de salud trabajan intensamente para poder informar sobre los mecanismos de transmisión, el espectro clínico de la enfermedad, los nuevos diagnósticos y las estrategias tanto de prevención, como de tratamiento potencial.

\section{Generalidades de la infección por SARS-CoV-2}

Al inicio, el nuevo virus fue llamado 2019-nCoV. Más tarde, la tarea de los expertos del Comité Internacional de Taxonomía de Virus (ICTV) lo denominó virus SARS-CoV-2, por la similitud al que causó el brote de SARS (SARS-CoV) en el año 2003, en China. En este contexto, resulta importante destacar la diferencia con el nombre de la enfermedad, la cual fue nombrada como COVID-19.

Los CoV son una gran familia de virus de ARN monocatenarios (+ssRNA) que pueden aislarse en diferentes especies animales. ${ }^{1}$ Se han convertido en los principales patógenos de brotes emergentes de enfermedades respiratorias y tienen el potencial de atravesar las barreras de las especies y causar en humanos enfermedades de diferente espectro de gravedad, los cuales van desde el resfriado común hasta los síndromes clínicos de MERS y SARS. Los últimos virus, probablemente, se originaron en murciélagos y luego se trasladaron a otros huéspedes mamíferos: la civeta de palma del Himalaya para SARS-CoV y el camello dromedario para MERS-CoV, antes de afectar a los humanos. La dinámica del SARS-Cov-2 se desconoce en este momento, pero se especula que también tiene un origen animal.

\section{Agente}

Los CoV son virus de ARN de cadena positiva con una apariencia de corona bajo un microscopio electrónico (coronam es el término en latín para corona), debido a la presencia de glucoproteínas de pico en la envoltura. La subfamilia Orthocoronavirinae de la familia Coronaviridae (orden Nidovirales) se clasifica en cuatro géneros de CoV: alphacoronavirus (alphaCoV), betacoronavirus (betaCoV), deltacoronavirus (deltaCoV) y gammacoronavirus (gammaCoV). Además, el género betaCoV se divide en cinco subgéneros o linajes. ${ }^{2}$ La caracterización genómica ha demostrado que, tal vez, los murciélagos y los roedores son las fuentes genéticas de alphaCoV y betaCoV. Por el contrario, las especies de aves parecen representar las fuentes genéticas de los deltaCoV y gammaCoV.

Los miembros de esta gran familia de virus pueden causar enfermedades a nivel respiratorio, entérico, hepático y en el sistema nervioso central. Hasta la fecha, se han identificado siete $\mathrm{CoV}$ humanos ( $\mathrm{HCoV}$ ) capaces de infectarlos.

En general, las estimaciones sugieren que el $2 \%$ de la población son portadores saludables de un $\mathrm{CoV}$ y que estos virus son responsables aproximadamente del 5 al 10\% de las infecciones respiratorias agudas. ${ }^{3}$

- CoV humanos comunes: HCoV-OC43 y HCoV-HKU1 (betaCoV del linaje A); HCoV-229E y HCoV-NL63 (alphaCoVs). Pueden causar resfriados comunes e infecciones autolimitadas de las vías respiratorias superiores en personas inmunocompetentes. En sujetos inmunocomprometidos y ancianos pueden ocurrir infecciones del tracto respiratorio inferior.

- Otros CoV humanos: SARS-CoV, SARS-CoV-2 y MERS$\mathrm{CoV}$ (betaCoV del linaje B y C, respectivamente). Son causantes de epidemias con gravedad clínica variable que presentan manifestaciones respiratorias y extrarrespiratorias. Con respecto al SARS-CoV, MERS-CoV, las tasas de mortalidad son de hasta 10 y $35 \%$, respectivamente.

El SARS-CoV-2 pertenece a la categoría betaCoVs. Tiene forma redonda o elíptica con diámetro de 60-140 nm, aproximadamente; es sensible a los rayos ultravioleta y al calor y puede ser inactivado efectivamente por solventes lipídicos que incluyen éter (75\%), etanol, desinfectante que contiene cloro, ácido peroxiacético y cloroformo, excepto la clorhexidina.

Chan et al. demostraron que el genoma del SARS-CoV-2 tenía un 89\% de identidad de nucleótidos con CoVZXC21 similar al SARS de murciélago y un $82 \%$ con el del SARSCoV humano. ${ }^{4}$ Su genoma de ARN monocatenario contiene 29,891 nucleótidos que codifican 9,860 aminoácidos.

Aunque sus orígenes no se han comprendido en su totalidad, probablemente evolucionó a partir de una cepa que se encuentra en los murciélagos. Se desconoce el posible huésped mamífero amplificador, intermedio entre murciélagos y humanos; dado que la mutación en la 
cepa original podría haber desencadenado directamente la virulencia hacia los humanos, no es seguro que este intermediario exista.

\section{Transmisión}

En sus inicios se presumió que la transmisión de animal a humano era el mecanismo principal, ya que los primeros casos estaban vinculados a la exposición directa al mercado mayorista de mariscos Huanan de Wuhan. Más tarde, se identificaron más casos que no se asociaron con este mecanismo de exposición. Hoy sabemos que el virus también puede transmitirse de persona a persona, y las personas sintomáticas son la fuente más frecuente de propagación del SARS-CoV-2. La posibilidad de transmisión antes de que aparezcan los síntomas parece ser poco frecuente, aunque no queda descartado; de esta manera, el aislamiento es la mejor manera de contener esta epidemia.

$\mathrm{Al}$ igual que con otros patógenos respiratorios como la gripe y el rinovirus, se cree que la transmisión se produce a través de las gotas respiratorias al toser y estornudar. La transmisión de aerosol también es posible en caso de exposición prolongada a concentraciones elevadas de éste en espacios cerrados.

Las investigaciones realizadas por los CDC de China y los CDC locales mencionan que el tiempo de incubación podría ser casi siempre de tres a siete días y hasta dos semanas, ya que el tiempo más largo desde la infección hasta los síntomas fue de 12.5 días $\left(95 \% \mathrm{Cl}, 9,2\right.$ a 18). ${ }^{5,6}$ Estos datos también mostraron que esta nueva epidemia se duplicó aproximadamente cada siete días, mientras que el número básico de reproducción (R0-R nada) es 2.2. En otras palabras, en promedio, cada paciente transmite la infección a 2.2 personas adicionales.

\section{Fisiopatología}

La fisiopatología y virulencia de los CoV y del SARS-CoV-2 tienen vínculos con la función de las proteínas estructurales. Su estructura contiene elementos que pueden bloquear la respuesta inmune innata del huésped. Entre las funciones de las proteínas estructurales, la envoltura tiene un papel crucial en la patogenicidad del virus, pues promueve el ensamblaje y la liberación viral.

Las glicoproteínas de espiga compuestas de dos subunidades (S1 y S2) están dentro de los elementos estructurales de los CoV. Los homotrímeros de proteínas $\mathrm{S}$ componen los picos en la superficie viral, guiando el enlace a los receptores del huésped. El dominio de unión al receptor, por el contrario, presenta sólo una identidad de aminoácidos de $40 \%$ con otros SARS-CoV. En el SARS-CoV-2, la subunidad S2, que contiene un péptido de fusión, un dominio transmembrana y un dominio citoplasmático, está altamente conservada, por lo que podría ser un objetivo para compuestos antivirales (anti-S2). ${ }^{7,8}$

\section{Cuadro clínico}

El espectro clínico de COVID-19 varía desde formas asintomáticas hasta afecciones clínicas caracterizadas por insuficiencia respiratoria, la cual requiere manejo con aporte de ventilación mecánica y estancia en una Unidad de Cuidados Intensivos (UCl), manifestaciones multiorgánicas y sistémicas en términos de sepsis, choque séptico y múltiples síndromes de disfunción orgánica (MODS) (Figura 1 y Tabla 1). En uno de los primeros informes sobre la enfermedad, Huang et al. ilustraron que los pacientes (n. 41) sufrían fiebre, malestar general, tos seca y disnea. ${ }^{9-11}$

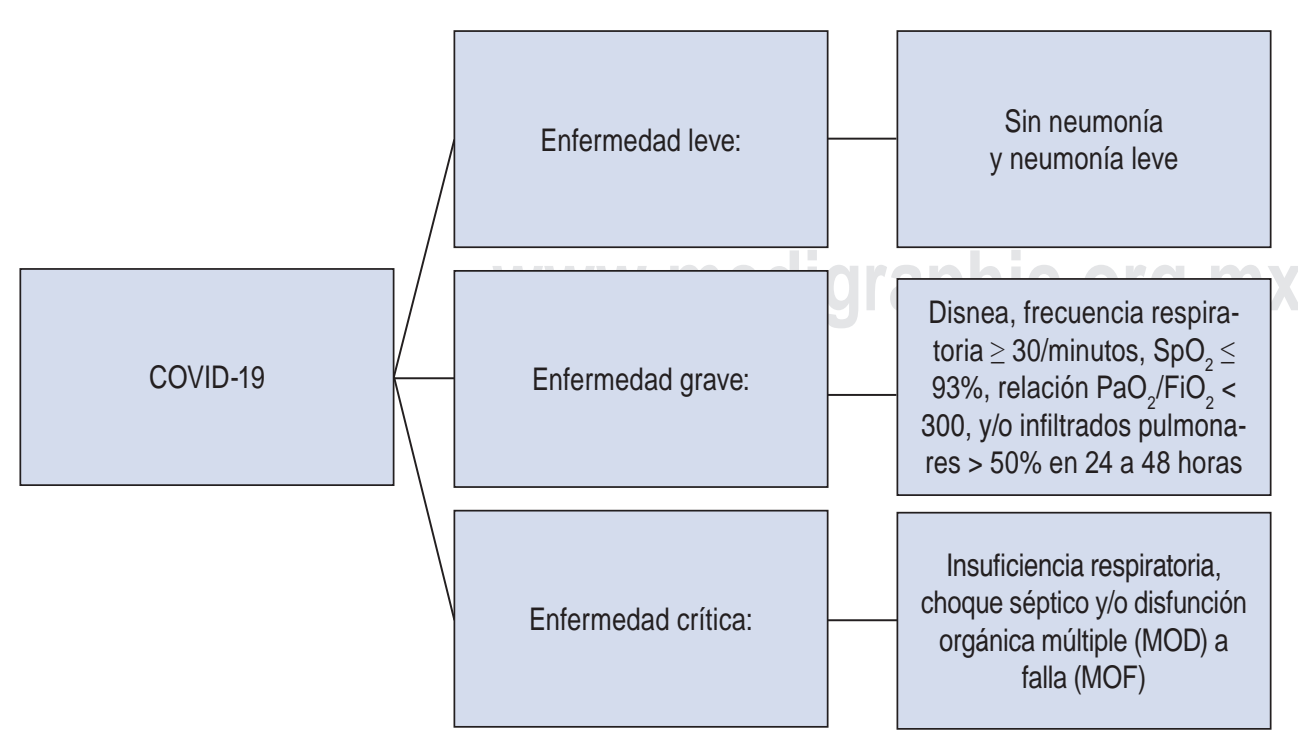

Figura 1:

Clasificación de síndromes clínicos en COVID-19.

$\mathrm{SpO}_{2}=$ saturación parcial de oxígeno en la sangre. $\mathrm{FiO}_{2}=$ fracción de oxígeno inspirado. 
Tabla 1: Características de síndromes clínicos de COVID-19.

\begin{tabular}{|l|l|l|}
\hline Enfermedad leve & Enfermedad moderada & Enfermedad grave \\
\hline $\begin{array}{l}\text { Síntomas de una infección viral de tracto respira- } \\
\text { torio superior, que incluye fiebre leve, tos (seca), } \\
\text { dolor de garganta, congestión nasal, dolor de } \\
\text { cabeza, dolor muscular o malestar general }\end{array}$ & $\begin{array}{l}\text { Síntomas respiratorios como tos y dificultad } \\
\text { para respirar (o taquipnea en niños) están } \\
\text { presentes sin signos de enfermedad severa }\end{array}$ & $\begin{array}{l}\text { Fiebre más disnea severa, dificultad respira- } \\
\text { toria, taquipnea (>30 respiraciones/min) e } \\
\text { hipoxia }\left(\mathrm{SpO}_{2}<90 \% \text { en el aire ambiente) }\right.\end{array}$ \\
& & La cianosis puede ocurrir en niños \\
\hline
\end{tabular}

El 29 de enero de 2020, Li et al. publicaron en New England Journal of Medicine (NEJM) los primeros 425 casos registrados en Wuhan. ${ }^{5}$ Dentro de los datos más relevantes se indicó que la edad media de los pacientes era de 59 años, sin diferencias significativas de género $(56 \%$ hombres). En el Journal of the American Medical Association, el 24 de febrero de 2020 se comunicaron los datos clínicos y epidemiológicos de los 72,314 registros de casos, incluyendo casos confirmados, sospechosos, diagnosticados y asintomáticos; en total fue $62 \%$ de casos confirmados, incluido el $1 \%$ de los casos que fueron asintomáticos, pero que fueron positivos en el laboratorio (prueba de ácido nucleico viral). Éste fue el primer acercamiento a una tasa de letalidad general de casos confirmados, la cual se estimó en el $2.3 \%$. En el análisis de las muertes registradas, se pudo identificar que fueron principalmente pacientes de edad avanzada, en particular aquéllos con edad $\geq 80$ años (alrededor del $15 \%$ ) y de 70 a 79 años (8.0\%), aproximadamente la mitad (49.0\%) de los pacientes críticos y afectados por comorbilidades preexistentes como enfermedades cardiovasculares, diabetes, enfermedades respiratorias crónicas y enfermedades oncológicas. En esta cohorte sólo el 1\% de los pacientes tenían menos de nueve años y en ellos no se registraron desenlaces fatales. ${ }^{12}$

Desde esos primeros reportes en China, los investigadores de los centros CDC decidieron dividir las manifestaciones clínicas de la enfermedad por su gravedad (Tabla 2).

\section{Estudios paraclínicos, imagen y serología}

Para efectuar el diagnóstico, la OMS recomienda recolectar muestras tanto del tracto respiratorio superior (muestras naso y orofaríngeas), como del tracto respiratorio inferior (esputo expectorado, aspirado endotraqueal o lavado broncoalveolar). La recolección de muestras de lavado broncoalveolar sólo debe realizarse en pacientes con ventilación mecánica; además éstas requieren almacenamiento a $4^{\circ}$ centígrados.

La amplificación del material genético extraído de la muestra de saliva o moco se realiza a través de una reacción en cadena de la polimerasa inversa (RT-PCR), que implica la síntesis de una molécula de ADN de doble cadena a partir de un molde de ARN, por lo que el primer paso deberá ser el de asegurar que el material genético sea suficiente. Las sondas utilizadas se basan en la secuencia génica inicial publicada por el Centro Clínico de Salud Pública de Shanghái y la Escuela de Salud Pública, Universidad de Fudan, Shanghái, China y la posterior evaluación confirmatoria por parte de laboratorios adicionales. Si el resultado de la prueba es positivo, se recomienda repetir la prueba para verificarla. En pacientes hospitalizados y aquéllos con enfermedad crítica y diagnóstico confirmado de COVID-19, la evaluación de laboratorio debe repetirse para evaluar el aclaramiento viral antes de ser liberado de la observación clínica.

En la etapa temprana de la enfermedad, por estudios paraclínicos se puede encontrar un recuento de glóbulos blancos totales normal o inclusive disminuido a expensas de baja cifra de linfocitos. La linfopenia parece ser un factor pronóstico negativo. Se pueden encontrar valores aumentados de enzimas hepáticas, LDH, enzimas musculares y proteína $\mathrm{C}$ reactiva. Hay un valor normal de procalcitonina. En pacientes críticos, el valor del dímero D aumenta, los linfocitos sanguíneos disminuyen de forma persistente y se encuentran alteraciones de laboratorio que tienden al desequilibrio multiorgánico (alta amilasa, trastornos de la coagulación, etcétera). ${ }^{13}$

Respecto a los hallazgos imagenológicos, Yoon et al. encontraron que $33.3 \%$ de su cohorte tenía anormalidades parenquimatosas detectadas por radiografía de tórax y la mayoría de las anormalidades eran consolidaciones periféricas. Las lesiones por tomografía de tórax mostraron afectación bilateral en más de $80 \%$ de los pacientes, además, se encontraron 77 lesiones pulmonares, incluidas lesiones irregulares (39\%), lesiones confluentes grandes (13\%) y lesiones nodulares pequeñas (48\%). Los segmentos pulmonares más periféricos y posteriores estuvieron involucrados en 78 y $67 \%$ de las lesiones, respectivamente. En general, las lesiones están típicamente mal definidas y están compuestas de opacidades mixtas de vidrio esmerilado y consolidación u opacidades puras de vidrio esmerilado, según describen los autores. Las lesiones parcheadas a confluentes se distribuyeron principalmente en los lóbulos inferiores y a lo largo de la pleura, mientras que las lesiones nodulares se distribuyeron principalmente a lo largo de los haces broncovasculares. ${ }^{14}$ 
$\mathrm{Xu}$ et al. en su investigación también reportaron que las presentaciones de imágenes de CT de NCP son en su mayoría opacidades irregulares de vidrio esmerilado en las áreas periféricas subpleural con consolidación parcial. ${ }^{15}$

Debido a que sólo 30\%, aproximadamente, de las lesiones son identificables por radiografía, la recomendación de realizar la tomografía computarizada proporcionará bases importantes para el diagnóstico temprano de la infección por COVID-19.

\section{Anatomía patológica}

Aunque se sabe poco de los aspectos anatomopatológicos de esta infección, Tian et al. informaron datos de histopatología obtenidos en los pulmones de dos pacientes que se sometieron a lobectomías pulmonares por adenocarcinoma. Los especímenes mostraron edema e importantes exudados proteicos como grandes glóbulos de proteína, congestión vascular combinada con grupos inflamatorios de material fibrinoide y células gigantes multinucleadas con hiperplasia de neumocitos. ${ }^{16}$

\section{Tratamiento}

No existe un tratamiento antiviral específico recomendado para COVID-19 y tampoco una vacuna disponi- ble. El tratamiento es sintomático y la oxigenoterapia representa la principal intervención de tratamiento para pacientes con infección grave. La ventilación mecánica puede ser necesaria en casos de insuficiencia respiratoria refractaria a la oxigenoterapia, mientras que el soporte hemodinámico es esencial para controlar el choque séptico (Tabla 3).

Entre otras estrategias terapéuticas, no se recomiendan los corticosteroides sistémicos para el tratamiento de la neumonía viral o el síndrome de dificultad respiratoria aguda (SDRA). Además, se debe evitar la administración no selectiva o inapropiada de antibióticos. Aunque no se han aprobado tratamientos antivirales se han sugerido alfainterferón (por ejemplo, cinco millones de unidades por inhalación de aerosol dos veces al día) y lopinavir/ritonavir. Los estudios preclínicos sugirieron que remdesivir (GS5734), un inhibidor de la ARN polimerasa con actividad in vitro contra múltiples virus de ARN, incluido el ébola, podría ser eficaz tanto para la profilaxis como para el tratamiento de las infecciones por SARS-CoV-2. Este fármaco se probó positivamente en un modelo de macaco Rhesus de infección por MERS-CoV.

La oxigenación por membrana extracorpórea (ECMO) para pacientes con hipoxemia refractaria, a pesar de la ventilación con protección pulmonar, debe ser considerada después de un análisis caso por caso. Asimismo, se puede

Tabla 2: Características de la enfermedad crítica en pacientes con COVID-19.

\begin{tabular}{|c|c|c|}
\hline Síndrome de dificultad respiratoria & Septicemia & Choque séptico \\
\hline
\end{tabular}


Tabla 3: Manejo invasivo de la vía aérea y metas de tratamiento en COVID-19 crítica.

\begin{tabular}{|c|c|}
\hline Ventilación mecánica invasiva & Ventilación mecánica no invasiva \\
\hline
\end{tabular}

sugerir la ventilación en posición prono para aquellos pacientes con malos resultados. ${ }^{17-19}$

Múltiples factores se han descrito como predictores de peor pronóstico o desenlaces mortales, entre ellos destacan la edad mayor a 65 años, la presencia de puntajes elevados de SOFA y dímero $D$ elevado al ingreso hospitalario. ${ }^{20-23}$

Asimismo, Yang et al. publicaron el 24 de febrero de 2020 un estudio observacional retrospectivo sobre el curso clínico de pacientes críticos con infección por SARS-CoV-2, en el cual incluyeron 52 pacientes; de éstos, la mayoría (51) presentaba enfermedad crónica y $32(61.5 \%)$ murieron a los 28 días. En sus resultados describieron como factores que se relacionaron con mayor mortalidad, la edad mayor de 65 años, comorbilidades y el desarrollo de SIRA. ${ }^{24}$

\section{Prevención}

Las medidas preventivas son la estrategia actual para limitar la propagación de casos. Debido a que una epidemia aumentará mientras R0 sea mayor que 1 (COVID-19 es 2.2), las medidas de control deben enfocarse en reducir el valor a menos de 1 .

Las estrategias preventivas se centran en el aislamiento de los pacientes y el control cuidadoso de la infección, incluidas las medidas apropiadas que deben adoptarse durante el diagnóstico y la prestación de atención clínica a un paciente infectado. Por ejemplo, debe adoptarse precauciones durante la recolección de muestras, y evitar el contacto con las gotas en el aire y debe evitarse la inducción de esputo.

La estrategia más importante es lavarse las manos con frecuencia y el uso de desinfectante de manos portátil, así como evitar el contacto con la cara y la boca después de interactuar con un entorno posiblemente contaminado (Figura 2).

Los trabajadores de la salud que atienden a personas infectadas deben utilizar precauciones de contacto en el aire, como máscaras N95 o FFP3, protección para los ojos, batas y guantes para evitar la transmisión del patógeno.

Los síntomas de las primeras etapas de la enfermedad son inespecíficos. El diagnóstico diferencial debe incluir la posibilidad de una amplia gama de trastornos respiratorios comunes infecciosos y no infecciosos (por ejemplo, vasculitis, dermatomiositis).

\section{Recomendaciones para pacientes con cáncer de pulmón avanzado}

Dado el estado inmunosupresor sistémico causado por malignidad y tratamientos contra el cáncer, los pacientes con cáncer avanzado (pulmonar u otro) pueden tener un mayor riesgo de infección y/o complicaciones derivadas de la misma enfermedad, por lo que es prioritario proponer recomendaciones hechas por expertos para el manejo óptimo de estos pacientes y distinguir los síntomas de probable infección por COVID-19 de potenciales efectos secundarios de los fármacos antitumorales.

Así, en todo paciente con cáncer avanzado con sospecha de COVID-19 se debe:

- Suspender cualquier tipo de tratamiento oncológico en un paciente previamente tratado con tratamiento antitumoral hasta tener la prueba que confirme o des- 
carte la infección (prueba serológica para SARS-CoV-2 por PCR tarda de 4-24 horas).

- No iniciar tratamientos antitumorales; éstos se deberán continuar de manera pendiente hasta tener la prueba que confirme o descarte COVID-19 (prueba serológica de PCR tarda de 4-24 horas).

En todo paciente con cáncer avanzado y con COVID-19 confirmada:

- Deberá mantenerse en vigilancia clínica durante 14 días y no deberá iniciarse tratamiento ni continuarse en el paciente previamente tratado. Las pautas dependerán de qué espectro de COVID-19 presente el paciente.

Si COVID-19 se encuentra dentro del espectro leve o moderado de la enfermedad, después de 14 días de vigilancia y mejoría clínica (paciente estable), cumpliendo aislamiento domiciliario, podrá iniciar su tratamiento oncológico. Si durante la vigilancia hay deterioro clínico seguir las pautas de enfermedad grave.

Si COVID-19 se encuentra dentro del espectro leve o moderado de la enfermedad, las pautas para el paciente previamente tratados al cumplir el aislamiento domiciliario serán:

- Reiniciar tratamiento de quimioterapia a los 14 días de mejoría clínica (se recomienda profilaxis primaria con G-CSF y considerar antimicrobiano para profilaxis primaria en el contexto de disminuir el potencial riesgo de complicaciones neumológicas infecciosas).

- Reiniciar radioterapia.

- Reiniciar inmunoterapia.

Si COVID-19 se encuentra dentro del espectro grave de la enfermedad en pacientes que aún no inician el tratamiento:

Paciente con COVID-19 que requirió manejo hospitalario fuera de UTI:

- Iniciar tratamiento oncológico con quimioterapia a los 14 días después del egreso (excepto agentes como bleomicina o similares con potencial de toxicidad pulmonar).

- Iniciar tratamiento con inmunoterapia, inhibidores de tirosina quinasa o radioterapia al encontrar el nadir de las pruebas funcionales respiratorias (a las 8-12 semanas del egreso con evaluación por DLCO y CVF al egreso y a las ocho a 12 semanas), excepto pacientes que ya recibían tratamiento con inmunoterapia o TKI con un mínimo de tres meses, ya que podrán reiniciar sus tratamientos desde las ocho semanas con riesgo bajo de neumonitis.

Paciente con COVID-19 requirió manejo hospitalario en UTI:

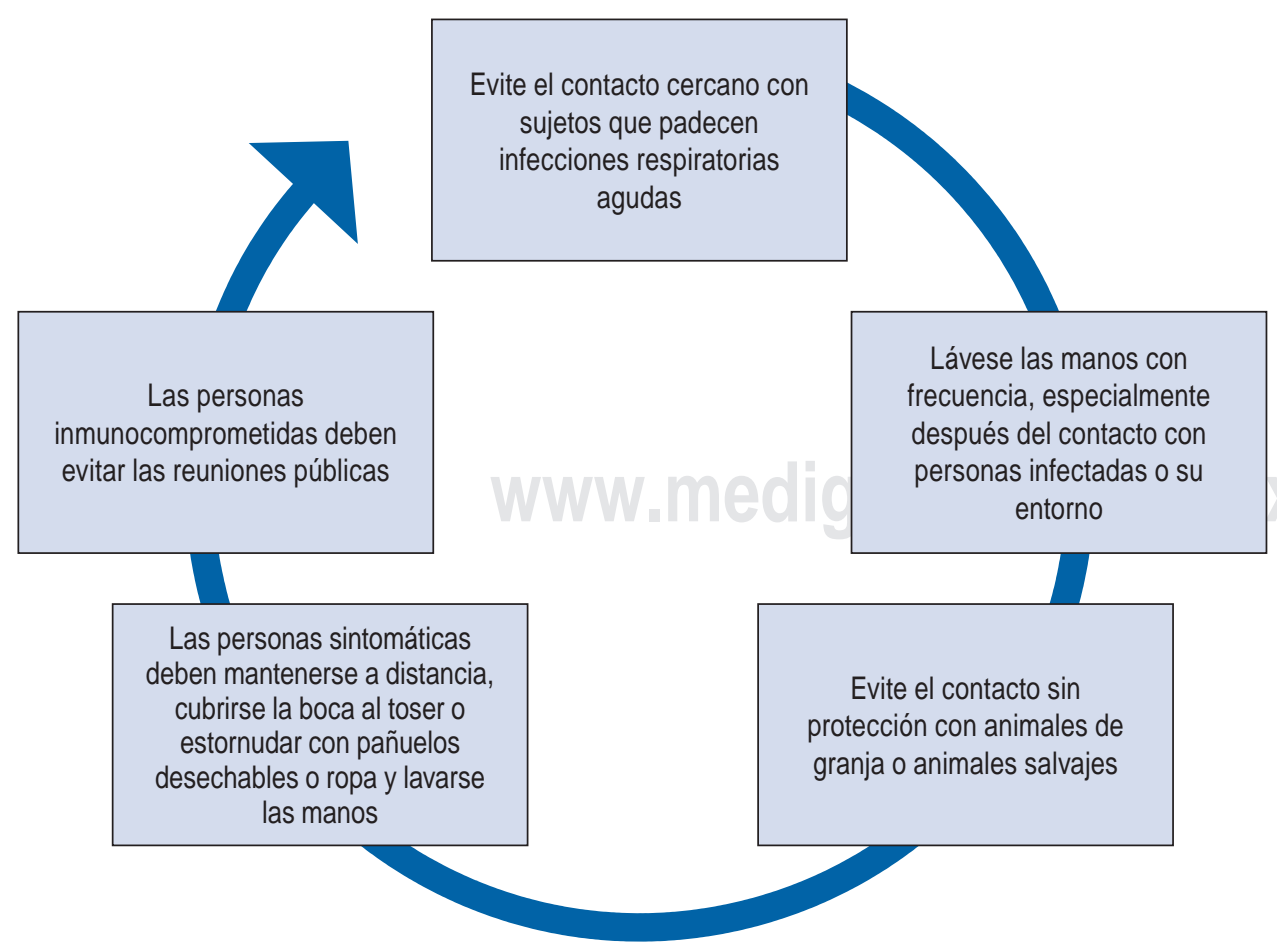

Figura 2:

Medidas de prevención para la infección de SARS-CoV-2. 
- Iniciar el tratamiento oncológico con quimioterapia a las ocho semanas del egreso (se recomienda profilaxis primaria con G-SCF y considerar antimicrobiano para profilaxis primaria en el contexto de disminuir el potencial riesgo de complicaciones neumológicas infecciosas).

- Iniciar el tratamiento con inmunoterapia o inhibidores de tirosina quinasa al encontrar el nadir de las pruebas funcionales respiratorias (a los tres meses del egreso mediante DLCO y CVF, las cuales se recomiendan tomar en tres tiempos: egreso, tres y seis meses) o considerar otra terapia oncológica.

- Iniciar el tratamiento de radioterapia con intento curativo a pulmón, al encontrar el nadir de las pruebas funcionales respiratorias, el cual es generalmente a los tres meses (DLCO y CVF se recomiendan tomar en tres tiempos: egreso, tres y seis meses). Si la radioterapia tiene la intención paliativa a pulmón deberá esperarse seis meses. La radioterapia a otros órganos que no abarquen pulmón o que la toxicidad no afecte al pulmón deberá iniciarse o continuar ocho a 12 semanas después, en especial si es con intento curativo. ${ }^{25}$

Si bien, no hay datos de la evolución de las pruebas de función respiratoria en pacientes con formas graves de COVID-19 al egreso, la evolución de estos pacientes infectados por influenza AH1N1 fue de estabilidad y mejoría de la función respiratoria hasta los seis meses. Zarogoulidis et al., ${ }^{25}$ en 2011, reportaron un nadir de la función respiratoria en las pruebas de función respiratoria (PFR) realizadas del tercer al sexto mes del egreso. Dentro de los parámetros de función pulmonar al egreso, comparados con los parámetros al tercer mes, se encontró que había ligera mejoría demostrada por CVF, pero en los parámetros a los seis meses ya no se documentó mejoría, lo cual traduce el mecanismo persistente de inflamación pulmonar a corto mediano plazo en los sobrevivientes; comparados con los parámetros de función pulmonar de personas sin evidencia de infección, ésta se ve gravemente comprometida. Estimamos una recuperación de función pulmonar similar en infectados por COVID-19, por lo que se hacen las recomendaciones previas cuando el paciente oncológico se encontraba o está por iniciar tratamientos con potencial de toxicidad pulmonar, ya sea o no inmunomediada.

\section{Pronóstico. Complicaciones y mortalidad}

Los datos preliminares sugieren que la tasa de mortalidad informada varía de 1 al 2\%, según el estudio y el país. La mayoría de las muertes han ocurrido en pacientes mayores de 50 años. Los niños parecen presentar sólo formas leves de la infección, pero pueden servir como vectores para una transmisión adicional. ${ }^{5}$
Las complicaciones a largo plazo entre los sobrevivientes de infección por SARS-CoV-2 con enfermedad clínicamente significativa de COVID-19 aún no están disponibles, pero se prevé que la reparación tisular a nivel pulmonar pueda generar secuelas de cicatrización (fibrosis) y que, de manera consecuente, éstas afecten la función pulmonar a largo plazo, demostrado por pruebas de mecánica e intercambio, tal como sucedió en caso de pacientes sobrevivientes en la pandemia de infección por influenza AH1N1. Las tasas de mortalidad para los casos a nivel mundial permanecen entre 1 y $2 \%$, aunque en pacientes con factores de riesgo para pronósticos desfavorables (comorbilidades diabetes mellitus, HAS, EPOC, edad avanzada con o sin cáncer pulmonar) no se han estimado cifras fidedignas de tasas de mortalidad hasta el día de hoy.

\section{CONCLUSIONES}

El nuevo coronavirus SARS-CoV-2 tiene diversas características epidemiológicas y biológicas. Las principales manifestaciones clínicas son similares a las de otras infecciones virales a nivel respiratorio, sin embargo, tiene el potencial de causar SDRA. Se han identificado factores predictores de pronósticos adversos, sin embargo, en pacientes con comorbilidad pulmonar aún faltan datos para identificar la tasa de letalidad, por lo que las medidas de prevención al día de hoy son prioritarias.

\section{REFERENCIAS}

1. Perlman S, Netland J. Coronaviruses pos-SARS: Update on replication and pathogenesis. Nat Rev Microbiol 2009;7(6):439-450. https://doi.org/10.1038/nrmicro2147.

2. Chan JF, To KK, Tse H, Jin DY, Yuen KY. Interspecies transmission and emergence of novel viruses: lessons from bats and birds. Trends Microbiol 2013;21(10):544-555. https://doi.org/10.1016/j.tim.2013.05.005.

3. Chen Y, Liu Q, Guo D. Emerging coronaviruses: Genome structure, replication, and pathogenesis. J Med Virol 2020;92(4):418-423. https:// doi.org/10.1002/jmv.25681.

4. Chan JF, Kok KH, Zhu Z, et al. Genomic characterization of the 2019 novel human-pathogenic coronavirus isolated from a patient with atypical pneumonia after visiting Wuhan. Emerg Microbes Infect 2020;9(1):221-236. https://doi.org/10.1080/22221751.2020.1719902.

5. Li Q, Guan X, Wu P, et al. Early transmission dynamics in Wuhan, China, of novel coronavirus-infected pneumonia. N Engl J Med 2020;382(13):1199-1207. https://doi.org/10.1056/nejmoa2001316.

6. Bauch ChT, Lloyd-Smith JO, Coffee MP, Galvani AP. Dynamically modeling SARS and other newly emerging respiratory illnesses: Past, present, and future. Epidemiology 2005;16(6):791-801. https:// doi.org/10.1097/01.ede.0000181633.80269.4c.

7. Song W, Gui M, Wang X, Xiang Y. Cryo-EM structure of the SARS coronavirus spike glycoprotein in complex with its host cell receptor ACE2. PLoS Pathog 2018;14(8):e1007236. https://doi.org/10.1371/ journal.ppat.1007236

8. Angeletti S, Benvenuto D, Bianchi M, Giovanetti M, Pascarella S, Ciccozzi M. COVID-2019: The role of the nsp2 and nsp3 in 
its pathogenesis. J Med Virol 2020;92(6):584-588. https://doi. org/10.1002/jmv.25719.

9. Zhu N, Zhang D, Wang W, et al. A novel coronavirus from patient with pneumoniain China, 2019. N Engl J Med 2020;382(8):727-733. https://doi.org/10.1056/nejmoa2001017.

10. Zhou P, Yang XL, Wang XG, et al. A pneumonia outbreak associated with a new coronavirus of probable bat origin. Nature 2020;579(7798):270-273.https://doi.org/10.1038/s41586-020-2012-7.

11. Huang Ch, Wang Y, Li X, et al. Clinical features of patients infected with 2019 novel coronavirus in Wuhan, China. Lancet 2020;395(10223):497506. https://doi.org/10.1016/s0140-6736(20)30183-5.

12. $\mathrm{Wu} Z$, McGoogan JM. Characteristics of and important lessons from the coronavirus disease 2019 (COVID-19) outbreak in China: Summary of a report of 72 ?314 cases from the Chinese Center for Disease Control and Prevention. JAMA 2020;323(13):1239-1242. https://doi.org/10.1001/jama.2020.2648.

13. Cascella M, Rajnik M, Cuomo A, Dulebohn SC, di Napoli R. Features, evaluation and treatment Coronavirus (COVID-19). Treasure Island (FL): StatPearls Publishing; 2020.

14. Yoon SH, Lee KH, Kim JY, et al. Chest radiographic and CT findings of the 2019 novel coronavirus disease (COVID-19): analysis of nine patients treated in Korea. Korean J Radiol 2020;21(4):494-500. https:// doi.org/10.3348/kjr.2020.0132.

15. $\mathrm{Xu} \mathrm{YH,} \mathrm{Dong} \mathrm{JH,} \mathrm{An} \mathrm{WM,} \mathrm{et} \mathrm{al.} \mathrm{Clinical} \mathrm{and} \mathrm{computed} \mathrm{tomography}$ imaging features of novel coronavirus pneumonia caused by SARS-CoV-2. J Infect 2020;80(4):394-400. https://doi.org/10.1016/j. jinf.2020.02.017.

16. Tian S, Hu W, Niu L, Liu H, Xu H, Xiao SY. Pulmonary pathology of early phase 2019 novel coronavirus (COVID-19) pneumonia in two patients with lung cancer. J Thorac Oncol 2020;15(5):700-704. https:// doi.org/10.1016/j.jtho.2020.02.010.

17. Hui DS, Chow BK, Lo T, et al. Exhaled air dispersion during high-flow nasal cannula therapy versus CPAP via different masks. Eur Respir J 2019;53(4):1802339. https://doi.org/10.1183/13993003.02339-2018.
18. Gordon CJ, Tchesnokov EP, Feng JY, Porter DP, Götte M. The antiviral compound Remdesivir potently inhibits RNA-dependent RNA polymerase from middle east respiratory syndrome coronavirus. J Biol Chem 2020;295(15):4773-4779. https://doi.org/10.1074/jbc.ac120.013056.

19. De Wit E, Feldmann F, Cronin J, et al. Prophylactic and therapeutic Remdesivir (GS-5734) treatment in the rhesus macaque model of MERS-CoV infection. Proc Natl Acad Sci USA 2020;117(12):67716776. https://doi.org/10.1073/pnas.1922083117.

20. Asociación Médica China, Rama de Oncología de la Asociación Médica China; Revista de la Asociación Médica China. Guía de Diagnóstico Clínico y Tratamiento del Cáncer de Pulmón de la Asociación Médica China (Edición 2018). Chinese J Oncology 2018:40(12):935964. https://doi.org/10.3760/cma.j.issn.0253-3766.2018.12.012.

21. Liang W, Guan W, Chen R, et al. Cancer patients in SARS-CoV-2 infection: a nationwide analysis in China. Lancet Oncol 2020;21(3):335337. https://doi.org/10.1016/s1470-2045(20)30096-6.

22. Guan W, Ni Z, Hu Y, et al. Clinical characteristics of 2019 novel coronavirus infection in China. New England Journal of Medicine 2020. doi: 10.1056/NEJMoa2002032

23. Zhou F, Yu T, Du R, et al. Clinical course and risk factors for mortality of adult inpatients with COVID-19 in Wuhan, China: a retrospective cohort study. Lancet 2020;395(10229):1054-1062. https://doi. org/10.1016/s0140-6736(20)30566-3.

24. Yang X, Yu Y, Xu J, et al. Clinical course and outcomes of critically ill patients with SARS-CoV-2 pneumonia in Wuhan, China: a singlecentered, retrospective, observational study. Lancet Respir Med 2020;8(5):475-481. Observational study. https://doi.org/10.1016/ s2213-2600(20)30079-5.

25. Zarogoulidis P, Kouliatsis G, Papanas N, et al. Long-term respiratory follow-up of H1N1 infection. Virol J 2011;8:319. https://doi. org/10.1186/1743-422x-8-319.

Conflicto de intereses: Los autores declaran no tener conflicto de intereses. 